\title{
THE HIGHER $K$-THEORY OF COMPLEX VARIETIES
}

\author{
Claudio Pedrini and Charles Weibel
}

March 28, 2000

\begin{abstract}
Let $X$ be a smooth complex variety, and let $F$ be its function field. We prove that (after localizing at the prime 2) the $K$-groups of $F$ are divisible above the dimension of $X$, and that the $K$-groups of $X$ are divisible-by-finite. We also describe the torsion in the $K$-groups of $F$ and $X$.
\end{abstract}

In this paper we shall describe the abelian group structure of the algebraic $K$-groups $K_{n}$ of a complex variety $X$ and its function field $F$, at least when $n>\operatorname{dim}(X)=\operatorname{tr}$. deg. $(F)$ and $K_{n}$ is localized at the prime $\ell=2$. Our results generalize Suslin's description of the $K$-theory of the complex numbers $\mathbb{C}$ in [Su1], and our earlier results for curves and surfaces in $[\mathrm{PW}]$.

Much of this paper depends upon the recent developments in motivic cohomology [SV,V2], related to the norm residue conjecture, that the the norm residue homomorphism $K_{n}^{M}(F) / m \rightarrow H_{\mathrm{et}}^{n}(F, \mathbb{Z} / m)$ is an isomorphism for all $m$; see [BK, p. 118]. Here $K_{*}^{M}(F)$ is Milnor $K$-theory and $H_{\text {et }}^{*}(F, \mathbb{Z} / m)$ is étale cohomology. Since this conjecture might be settled soon, we have been encouraged to state many of our results twice: first for $\ell=2$ and then under the assumption that the norm residue conjecture holds.

Our first main result, theorem A (3.2), is that the $\bmod 2^{\nu} K$-theory $K_{*}(F ; \mathbb{Z} / m)$ is isomorphic to the polynomial ring $H_{\mathrm{et}}^{*}(F, \mathbb{Z} / m)[\beta]$, where $\beta \in K_{2}(F ; \mathbb{Z} / m)$ and $m=2^{\nu}$ for any $\nu$. (This would hold for all $m$ if we knew the norm residue conjecture were true.) As observed by Suslin in [SuM] (see theorem 4.1), Theorem A implies that the Quillen-Lichtenbaum conjecture holds for smooth varieties over $\mathbb{C}$ (for all $m=2^{\nu}$ ).

Our second main result, theorem B (5.1), states that the groups $K_{n}(F)$ are 2-divisible for $n>d, d=\operatorname{tr}$. deg. $(F)$, and uses theorem A to describe the twoprimary torsion subgroup. If we assume the norm residue conjecture, then $K_{n}(F)$ is divisible for $n>d$, and theorem A completely describes its torsion subgroup.

Our third main result, theorem $\mathrm{C}(6.1)$, is that the groups $K_{n}(X)$ are the sum of a 2-divisible group and a finite group for $n>\operatorname{dim}(X)$. If $X$ is projective, we can improve this range to $n \geq \max \{1, \operatorname{dim}(X)-2\}$. If we assume the norm residue conjecture, then the groups $K_{n}(X)$ are divisible-by-finite in these ranges.

Theorem $\mathrm{C}$ generalizes our result in [PW] that if $X$ is a surface and $n>2$ then $K_{n}(X)$ is the sum of a divisible group and a finite group, which is either $H_{\text {an }}^{2}(X, \mathbb{Z})_{\text {tors }}$ or $H_{\text {an }}^{3}(X, \mathbb{Z})_{\text {tors }}$, according to the parity of $n$. An earlier indication of this phenomenon was given by the result of Colliot-Thélène and Raskind [CT-R, $2.2]$ that the finite group $H_{\mathrm{an}}^{3}(X, \mathbb{Z})_{\text {tors }}$ is a summand of $H^{1}\left(X, \mathcal{K}_{2}\right)$. 
To see why there is a restriction on $n$, observe that $K_{d}^{M}(F)$ is not divisible when $F$ is a function field $F$ of transcendence degree $d$. Indeed, if $E=\mathbb{C}\left(t_{1}, \ldots, t_{d}\right)$ is a purely transcendental subfield, then the iterated tame symbol and transfer $K_{d}^{M}(F) \rightarrow K_{d}^{M}(E) \rightarrow \mathbb{Z}$ is nonzero because it sends the symbol $\left\{t_{1}, \ldots, t_{d}\right\}$ to the integer $[F: E]$. On the other hand, if $n>d$ then the norm residue conjecture implies that $K_{n}^{M}(F)$ is divisible, because $H_{\mathrm{et}}^{n}(F, \mathbb{Z} / m)=0$. If $m=2^{\nu}$ then $K_{n}^{M}(F) / m \cong H_{\mathrm{et}}^{n}(F, \mathbb{Z} / m)$ by a theorem of Voevodsky [V2], so we at least know that the groups $K_{n}^{M}(F)$ are 2-divisible for $n>d$.

One way to interpret our results is to consider the comparison map $\rho$ between the algebraic $K$-theory of the variety $X$ and the topological $K$-theory $K U^{*}(X)$ of the underlying topological space. Defining the relative groups $K_{n}^{r e l}(X)$ to fit into an exact sequence

$$
\cdots K_{n+1}(X) \stackrel{\rho}{\rightarrow} K U^{-n-1}(X) \rightarrow K_{n}^{r e l}(X) \rightarrow K_{n}(X) \stackrel{\rho}{\rightarrow} K U^{-n}(X) \cdots,
$$

corollary 4.3 below states that $K_{n}^{r e l}(X)$ is uniquely 2-divisible (and conjecturally uniquely divisible) for all $n \geq \operatorname{dim}(X)-2$. Writing the finitely generated group $K U^{-n}(X)$ as $\mathbb{Z}^{r_{n}} \oplus B_{n}$, the torsion part $B_{n}$ comes from $K_{n}(X)$; by theorem $\mathrm{C}$, the free part $\mathbb{Z}^{r_{n+1}}$ of $K U^{-n-1}(X)$ is mirrored by a $(\mathbb{Q} / \mathbb{Z})^{r_{n+1}}$ in $K_{n}(X)$.

We have organized this paper as follows. In $\S 1$ we give some generalities about the norm residue conjecture and what it implies in our context. In $\S 2$ we give the additive structure of $K_{*}(F ; \mathbb{Z} / m)$, and the multiplicative structure in $\S 3$. We mention that the usual difficulty with products in $K_{*}(F ; \mathbb{Z} / 2)$ disappears because $\sqrt{-1} \in \mathbb{C}$; see $[\mathrm{AT}, 10.7]$. In $\S 4$ we recall how Suslin's argument in $[\mathrm{SuM}]$ yields the Quillen-Lichtenbaum Conjecture for smooth complex varieties, and use the relative groups $K_{*}^{r e l}(X)$ to draw some simple consequences. In $\S 5$ we use Deligne cohomology to prove theorem B, and in $\S 6$ we prove theorem $\mathrm{C}$. For this step we use Hodge theory and the sequence (4.2).

The results in this paper were first announced at the Colloque Karoubi in November 1998, before the announcement of [FS]. For this reason, our results do not invoke any multiplicative properties of the Bloch-Lichtenbaum spectral sequence for $F$, nor the generalized spectral sequence for $X$, both of which are established in [FS]. Even if we had used these more sophisticated tools, our proof of theorem A would be shortened only slightly (see remark 3.2.1), while the proofs of theorems $\mathrm{B}$ and $\mathrm{C}$ would not be affected at all.

It should be clear that although our use of Deligne cohomology requires working over $\mathbb{C}$, the method of Colliot-Thélène and Raskind [CT-R] works in the greater generality of schemes over a field with finite étale cohomological dimension. Some results in this greater generality, for $K_{*}(X ; \mathbb{Z} / m)$ and for $K_{*}$ of a surface, have been given by Levine in $\S 15$ of $[\mathrm{L}]$, using the constructions in [FS].

We will use the following notation. If $A$ is an abelian group and $m$ is a positive integer, ${ }_{m} A$ will denote the $m$-torsion subgroup of $A$, i.e., $\{a \in A: m a=0\}$, and we will write $A / m$ for $A / m A$. The torsion subgroup of $A$ will be written as $A_{\text {tors }}$, and its $\ell$-primary subgroup will be written as $A_{\ell \text {-tors }}$. If $\ell$ is a prime, then $\mathbb{Q}_{\ell} / \mathbb{Z}_{\ell}$ will denote the $\ell$-primary subgroup of $\mathbb{Q} / \mathbb{Z}$, i.e., the quotient of $\mathbb{Q}$ by the localization $\mathbb{Z}_{(\ell)}$ of $\mathbb{Z}$ at $\ell$. 


\section{$\S 1$. The Norm Residue Conjecture}

Let $F$ be a field. The Milnor $\operatorname{ring} K_{*}^{M}(F)$ is defined to be the quotient of the tensor algebra of the abelian group $F^{\times}$by the ideal generated by the quadratic Steinberg relations $\{x, 1-x\}$, where $x \in F^{\times}-\{1\}$. Thus $K_{0}^{M}(F)=\mathbb{Z}, K_{1}^{M}(F)=$ $F^{\times}$and $K_{2}^{M}(F) \cong K_{2}(F)$ by Matsumoto's theorem.

The norm residue homomorphism $F^{\times} \rightarrow H_{\mathrm{et}}^{1}\left(F, \mu_{m}\right)$ induces a cup product $F^{\times} \otimes F^{\times} \rightarrow H_{\mathrm{et}}^{2}\left(F, \mu_{m}^{\otimes 2}\right)$. Tate observed that this product satisfies the Steinberg relations. It follows that the norm residue map induces a ring homomorphism $K_{*}^{M}(F) / m \rightarrow H_{\mathrm{et}}^{*}\left(F, \mu_{m}^{\otimes *}\right)$.

We shall say that the norm residue conjecture holds for $m$ if the norm residue homomorphism $K_{*}^{M}(F) / m \rightarrow H_{\mathrm{et}}^{*}\left(F, \mu_{m}^{\otimes *}\right)$ is an isomorphism of graded rings. This was proven for all $m=2^{\nu}$ by Voevodsky in [V2], and is currently open for odd $m$. Since Milnor $K$-theory is presented by generators (in degree one) and relations (in degree two), this conjecture implies that the étale cohomology ring has the same presentation.

When $F$ contain $\mathbb{C}$, the root of unity $e^{2 \pi i / m}$ is a generator of the étale sheaf $\mu_{m}$, allowing us to identify $\mu_{m}$ with the constant sheaf $\mathbb{Z} / m$. This also identifies its twists $\mu_{m}^{\otimes *}$ with $\mathbb{Z} / m$, allowing us to consider the residue homomorphism as a morphism from $K_{*}^{M}(F) / m$ to $H_{\text {et }}^{*}(F, \mathbb{Z} / m)$.

Our proof of theorem A will be based upon the following result, due to Luca Barbieri-Viale [BV1, 4.3] [BV2, 2.2]; cf. [SV, 11.1] and [V2, 5.3]. We give the short proof here, for ease of reference. It is convenient to write $H_{\text {an }}^{n}(F, \mathbb{Z})$ for the direct limit of the classical (Betti) cohomology groups $H_{\text {an }}^{n}(U, \mathbb{Z})$ as $U$ runs over all Zariski open subsets of a variety $X$ with $F=\mathbb{C}(X)$.

Proposition 1.1. (Barbieri-Viale) Let $F$ be a field containing $\mathbb{C}$. Assume either that ( $i) \ell=2$ or (ii) the norm residue conjecture holds for $\ell$ and $F$. Then for each $n$ the group $H_{\mathrm{an}}^{n}(F, \mathbb{Z})$ has no $\ell$-torsion, and for each $m=\ell^{\nu}$ we have:

$$
K_{n}^{M}(F) / m \cong H_{\mathrm{et}}^{n}(F, \mathbb{Z} / m) \cong H_{\mathrm{an}}^{n}(F, \mathbb{Z}) \otimes \mathbb{Z} / m
$$

Proof. Since $H_{\mathrm{et}}^{n}(F, \mathbb{Z} / m)$ agrees with $H_{\mathrm{an}}^{n}(F, \mathbb{Z} / m)=\underline{\underline{\lim }} H_{\mathrm{an}}^{n}(U, \mathbb{Z} / m)$, we have exact sequences

$$
0 \rightarrow H_{\mathrm{an}}^{n}(F, \mathbb{Z}) \otimes \mathbb{Z} / m \rightarrow H_{\mathrm{et}}^{n}(F, \mathbb{Z} / m) \rightarrow{ }_{m} H_{\mathrm{an}}^{n+1}(F, \mathbb{Z}) \rightarrow 0 .
$$

Thus it suffices to show that the natural maps $H_{\mathrm{an}}^{n}(F, \mathbb{Z}) \rightarrow H_{\mathrm{et}}^{n}(F, \mathbb{Z} / m)$ are onto for any $n$ and $m=\ell^{\nu}$. Via the canonical map $H_{\text {an }}^{0}\left(U, \mathcal{O}_{X}^{\times}\right) \rightarrow H_{\text {an }}^{1}(U, \mathbb{Z})$ we get a canonical map $F^{\times} \rightarrow H_{\text {an }}^{1}(F, \mathbb{Z})$, lifting the residue map $F^{\times} / m \cong H_{\text {et }}^{1}(F, \mathbb{Z} / m)$. This establishes the case $n=1$. Now Tate observed that the cup product $H_{\text {et }}^{1}(F, \mathbb{Z} / m)^{\otimes n} \rightarrow H_{\text {et }}^{n}(F, \mathbb{Z} / m)$ factors through the norm residue homomorphism on $K_{n}^{M}(F) / m$. Thus we have a commutative square

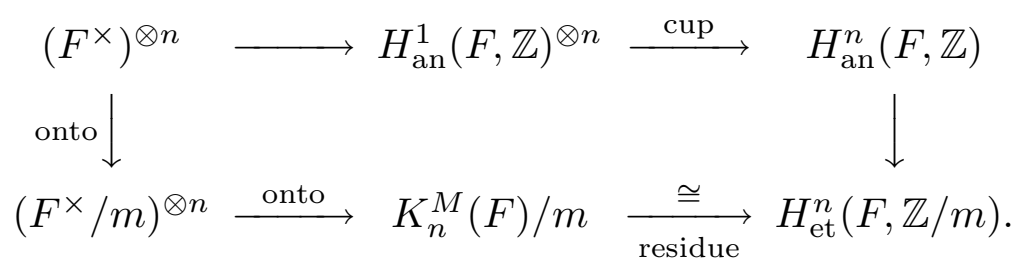


The bottom left map is onto by the presentation of $K_{n}^{M}(F)$, and the norm residue homomorphism is an isomorphism by Voevodsky's theorem (or by assumption if $m$ is odd). It follows that the right map is an surjection, as required.

Proposition 1.2. Let $F$ be a field containing $\mathbb{C}$. Each $H_{\mathrm{et}}^{n}\left(F, \mathbb{Q} / \mathbb{Z}_{2}\right)$ is a divisible group, and its $m$-torsion subgroup is $K_{n}^{M}(F) / m \cong H_{\mathrm{et}}^{n}(F, \mathbb{Z} / m)$ for all $m=2^{\nu}$.

Similarly, if the norm residue conjecture holds for $\ell$, then each $H_{\mathrm{et}}^{n}\left(F, \mathbb{Q}_{\ell} / \mathbb{Z}_{\ell}\right)$ is divisible, with

$$
H_{\mathrm{et}}^{n}\left(F, \mathbb{Q}_{\ell} / \mathbb{Z}_{\ell}\right) \cong K_{n}^{M}(F) \otimes \mathbb{Q}_{\ell} / \mathbb{Z}_{\ell} \cong H_{\mathrm{an}}^{n}(F, \mathbb{Z}) \otimes \mathbb{Q}_{\ell} / \mathbb{Z}_{\ell}
$$

and for all $m=\ell^{\nu}$ the $m$-torsion subgroup is $K_{n}^{M}(F) / m \cong H_{\mathrm{et}}^{n}(F, \mathbb{Z} / m)$.

Proof. Fix a prime $\ell$ (such as $\ell=2$ ) such that the norm residue conjecture holds for $\ell$. Divisibility follows from:

$$
H_{\mathrm{et}}^{n}\left(F, \mathbb{Q}_{\ell} / \mathbb{Z}_{\ell}\right)=\underline{\lim _{\mathrm{et}}} H_{\mathrm{et}}^{n}\left(F, \mathbb{Z} / \ell^{\nu}\right) \cong \varliminf_{\mathrm{lim}} K_{n}^{M}(F) / \ell^{\nu}=K_{n}^{M}(F) \otimes \mathbb{Q}_{\ell} / \mathbb{Z}_{\ell}
$$

Moreover, because there is no $\ell$-torsion in $H_{\mathrm{an}}^{n+1}(F, \mathbb{Z})$, the the Universal Coefficient Formula implies that $H_{\text {an }}^{n}\left(F, \mathbb{Q}_{\ell} / \mathbb{Z}_{\ell}\right)$ is isomorphic to $H_{\text {an }}^{n}(F, \mathbb{Z}) \otimes \mathbb{Q}_{\ell} / \mathbb{Z}_{\ell}$. To determine the $m$-torsion with $m=\ell^{\nu}$, we tensor the inclusion $\mathbb{Z} / m \subset \mathbb{Q}_{\ell} / \mathbb{Z}_{\ell}$ with the $\ell$-torsionfree group $H_{\text {an }}^{n}(F, \mathbb{Z})$, and invoke the isomorphism of $H_{\text {an }}^{n}(F, \mathbb{Z}) / m$ with $H_{\text {et }}^{n}(F, \mathbb{Z} / m)$ from 1.1 .

Example 1.2.1. For $m=\ell^{\nu}$ as above, we see that each group $H_{\mathrm{et}}^{n}(F, \mathbb{Z} / m)$ is free as a $\mathbb{Z} / m$-module. This is because the $m$-torsion of any divisible abelian group is free.

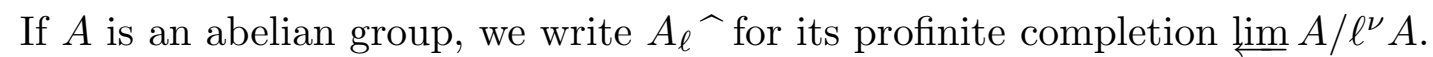

Corollary 1.3. Let $F$ be a field containing $\mathbb{C}$. Then the completion $K_{n}^{M}(F)_{2}$ has no 2-torsion for all $n$.

Similarly, if the norm residue conjecture holds for $\ell$, then $K_{n}^{M}(F) \widehat{\ell}$ is an $\ell$ torsionfree group for all $n$.

Proof. By 1.1, we have $K_{n}^{M}(F) / m \cong H_{\mathrm{an}}^{n}(F, \mathbb{Z}) / m$ for all $m=\ell^{\nu}$. Hence the $\ell$-adic completions of $K_{n}^{M}(F)$ and $H_{\text {an }}^{n}(F, \mathbb{Z})$ agree. But it is well known that the $\ell$-adic completion of an $\ell$-torsionfree group is $\ell$-torsionfree; see [AM, 10.3] for example.

We conclude this section with an analysis of $H_{\text {an }}^{*}\left(X, \mathbb{C}^{\times}\right)$which we will need in section 5. It is a generalization of Lemma 4.1 and 4.2 of [PW].

Lemma 1.4. Let $F=\mathbb{C}(X)$ be the field of rational functions of a smooth variety $X$ over $\mathbb{C}$. Assume either that (i) $\ell=2$ or (ii) the norm residue homomorphism holds for $\ell$. Then for all $j$ and $m=\ell^{\nu}$ :

(1) ${ }_{m} H_{\mathrm{an}}^{j}\left(F, \mathbb{C}^{\times}\right) \cong H_{\mathrm{et}}^{j}(F, \mathbb{Z} / m)$;

(2) $H_{\mathrm{an}}^{j}\left(F, \mathbb{C}^{\times}\right)$is $\ell$-divisible, and its $\ell$-primary torsion subgroup is

$$
H_{\mathrm{an}}^{j}\left(F, \mathbb{C}^{\times}\right)_{\ell \text {-tors }} \cong H_{\mathrm{et}}^{j}\left(F, \mathbb{Q}_{\ell} / \mathbb{Z}_{\ell}\right) \cong H_{\mathrm{an}}^{j}(F, \mathbb{Z}) \otimes \mathbb{Q}_{\ell} / \mathbb{Z}_{\ell}
$$


(3) If $A_{j}$ denotes the finite group $H_{\text {an }}^{j}(X, \mathbb{Z})_{\text {tors }}$, then

$$
H^{j}(X, \mathbb{Z} / m) \cong{ }_{m} H_{\text {an }}^{j}\left(X, \mathbb{C}^{\times}\right) \oplus A_{j} / m
$$

(4) $H^{j}\left(X, \mathbb{Q}_{\ell} / \mathbb{Z}_{\ell}\right) \cong H_{\text {an }}^{j}\left(X, \mathbb{C}^{\times}\right)_{\ell \text {-tors }}$

Proof. From the exponential sequence, and the fact that $H_{\text {an }}^{j}(X, \mathbb{C})$ is uniquely divisible, it follows that $H_{\mathrm{an}}^{j-1}\left(X, \mathbb{C}^{\times}\right)$is the sum of a divisible group and the finite group $A_{j}=H_{\text {an }}^{j}(X, \mathbb{Z})_{\text {tors }}$. Replacing $X$ by open subsets $U$ and passing to the limit, the fact that ${ }_{\ell} H_{\text {an }}^{j}(F, \mathbb{Z})=0$ (see 1.1) implies that $H_{\text {an }}^{j}\left(F, \mathbb{C}^{\times}\right)$is $\ell$-divisible.

Using the Kummer sequence for $0 \rightarrow \mathbb{Z} / m \rightarrow \mathbb{C}^{\times} \stackrel{m}{\longrightarrow} \mathbb{C}^{\times} \rightarrow 0$, this implies part $(1)$, that $H^{j}(F, \mathbb{Z} / m) \cong{ }_{m} H_{\text {an }}^{j}\left(F, \mathbb{C}^{\times}\right)$, and that $H^{j}(X, \mathbb{Z} / m)$ is an extension of ${ }_{m} H_{\text {an }}^{j}\left(X, \mathbb{C}^{\times}\right)$by $A_{j} / m \cong H_{\text {an }}^{j-1}\left(X, \mathbb{C}^{\times}\right) / m$. To prove $(3)$, that the extension splits, we need to observe that the inclusion $A_{j} / m \rightarrow H_{\text {an }}^{j}(X, \mathbb{Z} / m)$ factors as

$$
A_{j} / m \cong H_{\mathrm{an}}^{j-1}\left(X, \mathbb{C}^{\times}\right) / m \stackrel{\text { summand }}{\longrightarrow} H_{\mathrm{an}}^{j}(X, \mathbb{Z}) / m \stackrel{\text { summand }}{\longrightarrow} H_{\mathrm{an}}^{j}(X, \mathbb{Z} / m),
$$

where the final map is a split inclusion by the universal coefficient theorem. Passing to the limit over $m$ yields (2) and (4).

We can also show that the $\ell$-adic cohomology $H^{n}\left(X, \mathbb{Z}_{\ell}\right)=\varliminf^{n} H^{n}\left(X, \mathbb{Z} / \ell^{\nu}\right)$ is the sum of a torsionfree group and the finite $\ell$-primary torsion subgroup $\left(A_{n}\right)_{\ell \text {-tors }}$ of $H_{\mathrm{an}}^{n}(X, \mathbb{Z})$.

Recall that the $\ell$-adic Tate module $T_{\ell}(H)$ of an abelian group $H$ is the inverse limit of the system of groups $\ell^{\nu} H$.

$$
T_{\ell}(H)=\varliminf_{\ell}\left\{H \leftarrow \ell^{2} H \leftarrow \cdots \leftarrow \ell^{\nu} H \leftarrow \cdots\right\}
$$

It is well known that the Tate module is always torsionfree (see [CT-R, 1.3]).

Corollary 1.5. Let $\ell$ be either 2 , or a prime for which the norm residue conjecture holds. Then $H^{n}\left(X, \mathbb{Z}_{\ell}\right)$ is the direct sum of the torsionfree group $T_{\ell}\left(H_{\text {an }}^{n}\left(F, \mathbb{C}^{\times}\right)\right)$ and the finite group $\left(A_{n}\right)_{\ell \text {-tors }}$.

Proof. By Lemma 1.4, $H^{n}\left(X, \mathbb{Z}_{\ell}\right)$ is the inverse limit of the tower of groups $H^{n}\left(X, \mathbb{Z} / \ell^{\nu}\right) \cong{ }_{\ell^{\nu}} H_{\text {an }}^{n}\left(X, \mathbb{C}^{\times}\right) \oplus A_{n} / \ell^{\nu}$. That is, it is an extension of the Tate module of $H_{\mathrm{an}}^{n}\left(X, \mathbb{C}^{\times}\right)$, which is torsionfree, by the $\ell$-primary group $\left(A_{n}\right)_{\ell \text {-tors }}$. 
§2. The Additive structure of $K_{*}(F ; \mathbb{Z} / m)$.

The goal of this section is to prove the additive part of theorem A. This involves the groups $K_{n}(F ; \mathbb{Z} / m)$, which fit into a universal coefficient sequence:

$$
0 \rightarrow K_{n}(F) / m \rightarrow K_{n}(F ; \mathbb{Z} / m) \rightarrow{ }_{m} K_{n-1}(F) \rightarrow 0 .
$$

When $n=2$ for example, we have ${ }_{m} K_{1}(F)=\mu_{m}(F)$ and $K_{2}(F) / m \cong H_{\text {et }}^{2}\left(F, \mu_{m}^{\otimes 2}\right)$ for every $m$ by the Merkurjev-Suslin theorem [MS]. If $\mathbb{C} \subseteq F$ then the inclusion of $\mathbb{Z} / m \cong K_{2}(\mathbb{C} ; \mathbb{Z} / m)$ is split by $K_{2}(F ; \mathbb{Z} / m) \rightarrow \mu_{m}(F)$. Thus $K_{2}(F ; \mathbb{Z} / m) \cong$ $K_{2}(F) / m \oplus \mathbb{Z} / m$, where the summand $\mathbb{Z} / m$ is called the Bott summand, and is generated by the Bott element $\beta$, corresponding to the root of unity $\zeta_{m}=e^{2 \pi i / m}$.

For the sake of uniform exposition, we shall work with a prime $\ell$ such that the norm residue homomorphism holds for $\ell$ (and hence for all powers of $\ell$ ). In particular, the reader may take $\ell=2$ throughout.

We begin with a study of the groups $C H^{i}\left(F, n ; \mathbb{Q}_{\ell} / \mathbb{Z}_{\ell}\right)$. These are the direct limits of the groups $C H^{i}\left(F, n ; \mathbb{Z} / \ell^{\nu}\right)$ as $\nu \rightarrow \infty$.

Lemma 2.1. Assume that the norm residue conjecture holds for $\ell$ (e.g., $\ell=2)$. Then the groups $C H^{i}\left(F, n ; \mathbb{Q}_{\ell} / \mathbb{Z}_{\ell}\right)$ are divisible.

Proof. Suslin has proven that $C H^{i}(F, n) \cong H_{M}^{2 i-n}(F, \mathbb{Z}(i))$ for all $i$ and $n$, where $H_{M}$ denotes motivic cohomology; see [Su3] and [FV], [V1] (cf. [W, 4.2]). Using [V2], we have:

$$
C H^{i}\left(F, n ; \mathbb{Q}_{\ell} / \mathbb{Z}_{\ell}\right) \cong H_{M}^{2 i-n}\left(F, \mathbb{Q}_{\ell} / \mathbb{Z}_{\ell}(i)\right) \cong H_{\mathrm{et}}^{2 i-n}\left(F, \mathbb{Q}_{\ell} / \mathbb{Z}_{\ell}\right) .
$$

These groups are divisible by proposition 1.2.

The Bloch-Lichtenbaum spectral sequence [BL] has an analogue with coefficients (constructed in $[\mathrm{RW}]$ and $[\mathrm{FS}]$ ), starting from the higher Chow groups of [Bl]:

$$
E_{2}^{p q}=C H^{-q}\left(F,-p-q ; \mathbb{Q}_{\ell} / \mathbb{Z}_{\ell}\right) \Longrightarrow K_{-p-q}\left(F ; \mathbb{Q}_{\ell} / \mathbb{Z}_{\ell}\right)
$$

This is the direct limit of similar spectral sequences with coefficients $\mathbb{Z} / \ell^{\nu}$.

Operations 2.3. Now Soulé has shown in [Sou3] [GS, 7.1] that the Adams operations $\psi^{k}$ act naturally on this spectral sequence, commuting with the differentials, and that $\psi^{k}=k^{i}$ on the row $q=-i$. We deduce two facts from this.

First of all, by [Sou2, 2.8], for each $r$ there is an integer $M$, independent of $p$ and $q$, so that $M \cdot d_{r}^{p q}=0$. That is, the image of each differential has bounded exponent. In the case at hand, when the $E_{2}^{p q}$ are divisible groups, this forces the differentials $d_{2}^{p q}$ to be zero. By induction, the $E_{r}^{p q}$ are divisible and the differentials $d_{r}^{p q}$ are zero for all $r$, i.e., the spectral sequence converging to $K_{*}\left(F ; \mathbb{Q}_{\ell} / \mathbb{Z}_{\ell}\right)$ must degenerate at $E_{2}$.

In the second place, the same divisibility granted above allows us to construct idempotent operators on the spectral sequence. Fix $N \gg 0$ and $k$ and define

$$
f_{i}(t)=\prod_{\substack{j=1 \\ i \neq j}}^{N}\left(t-k^{j}\right), \quad c_{i}=f_{i}(k)=\prod\left(k^{i}-k^{j}\right) .
$$


Then $f_{i}\left(\psi^{k}\right)$ acts on the spectral sequence, is multiplication by $c_{i}$ on the row $q=$ $-i$, and vanishes on all other rows with $q \geq-N$. If $x$ is in $E_{r}^{p q}$ or $K_{n}\left(F, \mathbb{Q}_{\ell} / \mathbb{Z}_{\ell}\right)$, we define $e_{i}(x)$ to be $f_{i}\left(\psi^{k}\right)(y)$, where $y$ is such that $c_{i} y=x$. It is easy to see that $e_{i}$ is a well-defined operator on the spectral sequence, satisfying $e_{i}=e_{i}^{2}$. Indeed, $e_{i}$ is the identity on the row $q=-i$ and is zero elsewhere. Since $e_{i}$ acts on the filtration for $K_{n}\left(F ; \mathbb{Q}_{\ell} / \mathbb{Z}_{\ell}\right)$, it follows that the image of the operator $e_{i}$ on $K_{n}\left(F ; \mathbb{Q}_{\ell} / \mathbb{Z}_{\ell}\right)$ is a summand isomorphic to $C H^{i}\left(F, n ; \mathbb{Q}_{\ell} / \mathbb{Z}_{\ell}\right) \cong H_{\text {et }}^{2 i-n}\left(F, \mathbb{Q}_{\ell} / \mathbb{Z}_{\ell}\right)$.

Proposition 2.4. Let $F$ be a field containing $\mathbb{C}$. Assume either that $(i) \ell=2$ or (ii) the norm residue homomorphism holds for $\ell$. Then $K_{n}\left(F ; \mathbb{Q}_{\ell} / \mathbb{Z}_{\ell}\right)$ is a divisible group for all $n$. Moreover, there is a canonical isomorphism for all $m=\ell^{\nu}$ :

$$
\begin{aligned}
K_{n}\left(F ; \mathbb{Q}_{\ell} / \mathbb{Z}_{\ell}\right) \cong \bigoplus_{n / 2 \leq i \leq n} C H^{i}\left(F, n ; \mathbb{Q}_{\ell} / \mathbb{Z}_{\ell}\right) \cong \bigoplus_{j=0}^{[n / 2]} H_{\mathrm{et}}^{n-2 j}\left(F, \mathbb{Q}_{\ell} / \mathbb{Z}_{\ell}\right) ; \\
K_{n}(F ; \mathbb{Z} / m) \cong \bigoplus_{n / 2 \leq i \leq n} C H^{i}(F, n ; \mathbb{Z} / m) \cong \bigoplus_{j=0}^{[n / 2]} H_{\mathrm{et}}^{n-2 j}(F, \mathbb{Z} / m) .
\end{aligned}
$$

Proof. Our analysis of the Adams operations in 2.3 shows that the spectral sequence $(2.2)$ degenerates at $E_{2}$, and each $K_{n}\left(F ; \mathbb{Q}_{\ell} / \mathbb{Z}_{\ell}\right)$ is canonically a direct sum of the $C H^{i}\left(F, n ; \mathbb{Q}_{\ell} / \mathbb{Z}_{\ell}\right)$. The indexing in the statement of 2.4 uses $j=n-i$.

The coefficient sequence $0 \rightarrow \mathbb{Z} / m \rightarrow \mathbb{Q}_{\ell} / \mathbb{Z}_{\ell} \stackrel{m}{\longrightarrow} \mathbb{Q}_{\ell} / \mathbb{Z}_{\ell} \rightarrow 0$, together with the divisibility of the groups $K_{n}\left(F ; \mathbb{Q}_{\ell} / \mathbb{Z}_{\ell}\right)$, shows that $K_{n}(F ; \mathbb{Z} / m)$ is the $m$-torsion subgroup of $K_{n}\left(F ; \mathbb{Q}_{\ell} / \mathbb{Z}_{\ell}\right)$. Its description follows from proposition 1.2.

Example 2.4.1. When $n=2, K_{2}(F ; \mathbb{Q} / \mathbb{Z})$ is the direct sum of the Bott summand $\mathbb{Q} / \mathbb{Z}=H^{0}(F, \mathbb{Q} / \mathbb{Z})$ and the Brauer group $\operatorname{Br}(F) \cong K_{2}(F) \otimes \mathbb{Q} / \mathbb{Z} \cong H^{2}(F, \mathbb{Q} / \mathbb{Z})$.

This proves the additive half of theorem A. In order to analyse the ring structure, we first turn to étale $K$-theory of $F$. Recall from [DF, 5.2] that there is a right half-plane Atiyah-Hirzebruch spectral sequence:

$$
E_{2}^{p q}=\left\{\begin{array}{ll}
H_{\mathrm{et}}^{p}\left(F, \mathbb{Q}_{\ell} / \mathbb{Z}_{\ell}\right) & q \text { even } \\
0 & q \text { odd }
\end{array}\right\} \Longrightarrow K_{-p-q}^{e t}\left(F ; \mathbb{Q}_{\ell} / \mathbb{Z}_{\ell}\right)
$$

Proposition 2.5. Let $F$ be any field over $\mathbb{C}$. Assume either that (i) $\ell=2$ or (ii) the norm residue homomorphism holds for $\ell$.

Then $K_{n}^{e t}\left(F ; \mathbb{Q}_{\ell} / \mathbb{Z}_{\ell}\right)$ is a divisible group for all $n$, and the Atiyah-Hirzebruch spectral sequence (2.5.0) degenerates at $E_{2}$ to yield:

$$
K_{n}^{e t}\left(F ; \mathbb{Q}_{\ell} / \mathbb{Z}_{\ell}\right) \cong \bigoplus_{\substack{p \equiv n \\(\bmod 2)}} H^{p}\left(F, \mathbb{Q}_{\ell} / \mathbb{Z}_{\ell}\right) ; \quad K_{n}^{e t}\left(F ; \mathbb{Z} / \ell^{\nu}\right) \cong \bigoplus_{\substack{p \equiv n \\(\bmod 2)}} H^{p}\left(F, \mathbb{Z} / \ell^{\nu}\right)
$$

Proof. The proof for étale $K$-theory is similar to the proof of proposition 2.4 , using proposition 1.2, and goes back to Thomason [T, 4.3]. Here we use the action of the Adams operations on the Atiyah-Hirzebruch spectral sequence (2.5.0), exactly as in 2.3 , to see that it also degenerates. 


\section{§3. THE RING $K_{*}(F, \mathbb{Z} / m)$}

In this section we shall state theorem $\mathrm{A}$ and deduce some simple consequences for the ring $K_{*}(F ; \mathbb{Z} / m)$ and its cousin, the étale $K$-theory $K_{*}^{e t}(F ; \mathbb{Z} / m)$ of $F$ with coefficients $\mathbb{Z} / m$.

We begin with a description of the étale $K$-groups $K_{*}^{e t}(F ; \mathbb{Z} / m)$. Recall that there are natural maps $\rho_{n}(X): K_{n}(X ; \mathbb{Z} / m) \rightarrow K_{n}^{e t}(X ; \mathbb{Z} / m)$ for any scheme $X$, constructed in $[\mathrm{Fr} 2,1.3]$. Thomason proved in $[\mathrm{T}, 4.1]$ that $\rho(X)$ induces an isomorphism $K_{*}^{\text {et }}(X ; \mathbb{Z} / m) \cong K_{*}(X ; \mathbb{Z} / m)\left[\beta^{-1}\right]$, at least in our case when $X$ is a smooth variety over $\mathbb{C}$. (See [DFST] when $m$ isn't divisible by 2 or 3 .)

For every field $F$ the composition of $K_{n}^{M}(F) \rightarrow K_{n}(F ; \mathbb{Z} / m)$ with $\rho_{n}(F)$ induces a map $\tau_{n}: K_{n}^{M}(F) \rightarrow K_{n}^{e t}(F ; \mathbb{Z} / m)$.

Theorem 3.1. Let $F$ be any field over $\mathbb{C}$. Assume either that (i) $m=2^{\nu}$ or (ii) the norm residue conjecture holds for $m$. Then $\tau: K_{*}^{M}(F) \rightarrow K_{*}^{\text {et }}(X ; \mathbb{Z} / m)$ induces a graded ring isomorphism

$$
K_{*}^{e t}(F ; \mathbb{Z} / m) \cong K_{*}^{M}(F) / m\left[\beta, \beta^{-1}\right] \cong H_{\mathrm{et}}^{*}(F ; \mathbb{Z} / m)\left[\beta, \beta^{-1}\right] .
$$

Proof. The map $F^{\times} / m \rightarrow K_{1}^{\text {et }}(F ; \mathbb{Z} / m)$ induces a graded ring homomorphism from $K_{*}^{M}(F) / m[\beta]$ to $K_{*}^{e t}(F ; \mathbb{Z} / m)$. In terms of the filtration coming from the analogue of $(2.5 .0)$ using coefficients $\mathbb{Z} / m$, there is an isomorphism of $E_{2}^{12}=$ $H_{\text {et }}^{1}\left(F, \mu_{m}\right)$ with $F^{\times} / F^{\times m}$, and the Bott element $\beta \in E_{2}^{02}$ induces an isomorphism between $E_{2}^{02}=H_{\mathrm{et}}^{0}\left(F, \mu_{m}\right)$ and $\mathbb{Z} / m$. Together, they induce a bigraded ring isomorphism $H_{\text {et }}^{*}(F, \mathbb{Z} / m)\left[\beta, \beta^{-1}\right] \stackrel{\cong}{\longrightarrow} E_{2}^{* *}$ by $[\mathrm{DF}, 5.4]$ if $\ell^{\nu} \neq 2$. But the spectral sequence degenerates by 2.5 , and this isomorphism is compatible with the abutment $\operatorname{map} K_{*}^{M}(F) / m\left[\beta, \beta^{-1}\right]$ to $K_{*}^{e t}(F ; \mathbb{Z} / m)$, again by [DF, 5.4]. The result follows.

Theorem A (3.2). Let $F$ be a field of transcendence degree $d$ over $\mathbb{C}$. Assume either that ( $i) m=2^{\nu}$ or (ii) the norm residue conjecture holds for $m$. Then

(1) The Bott element $\beta$ is a nonzerodivisor in the ring $K_{*}(F ; \mathbb{Z} / m)$.

(2) $K_{*}(F ; \mathbb{Z} / m)$ is a polynomial ring over $K_{*}^{M}(F) / m$, where the indeterminate $\beta$ in $K_{2}(F ; \mathbb{Z} / m)$ is the Bott element. That is, there is a graded ring isomorphism:

$$
K_{*}(F ; \mathbb{Z} / m) \cong K_{*}^{M}(F) / m[\beta] \cong H_{\mathrm{et}}^{*}(F, \mathbb{Z} / m)[\beta]
$$

(3) the natural maps $\rho_{n}(F): K_{n}(F ; \mathbb{Z} / m) \rightarrow K_{n}^{\text {et }}(F ; \mathbb{Z} / m)$ are injections for all $n$, and isomorphisms for $n \geq d-1$. Thus for all $n \geq d-1$ we have a canonical multiplicative decomposition:

$$
K_{n}(F ; \mathbb{Z} / m) \cong \begin{cases}\oplus_{i=0}^{[d / 2]} H_{\mathrm{et}}^{2 i}(F, \mathbb{Z} / m) & \text { if } n \text { is even } \\ \bigoplus_{i=0}^{[d / 2]} H_{\mathrm{et}}^{2 i+1}(F, \mathbb{Z} / m) & \text { if } n \text { is odd }\end{cases}
$$


Remark 3.2.1. For tr. deg. $(F) \leq 2$, part (3) was announced by Suslin in [SuM, p. 350]; a proof may be found in [PW, 2.1]. Suslin's proof in loc. cit. assumes multiplicative properties of the Bott element $\beta \in K_{2}(\mathbb{C} ; \mathbb{Z} / m)$ on the BlochLichtenbaum spectral sequence, properties which were then unknown. (These properties are now consequences of our theorem A, or of [FS].)

Lemma 3.3. Let $F$ be a function field $F=E(s)$ over a field $E$, where $s$ is an indeterminate. Assume that the $\operatorname{map} K_{*}^{M}(k) / m[\beta] \rightarrow K_{*}(k ; \mathbb{Z} / m)$ is an isomorphism for all finite extensions $k$ of $E$. Then the map $K_{*}^{M}(F) / m[\beta] \rightarrow K_{*}(F ; \mathbb{Z} / m)$ is also an isomorphism.

Proof. We regard $F=E(s)$ as the field of fractions of the polynomial ring $R=E[s]$. For every residue field $k=E[s] / \wp$ of $E[s]$, there is a tame symbol $\partial_{k}: K_{n}(F ; \mathbb{Z} / m) \rightarrow K_{n-1}(k ; \mathbb{Z} / m)$, and the direct sum over all such $k$ is one of the maps in the $K$-theory localization sequence. It is well-known (see [Sou1, p.271]) that the $K$-theory localization sequence breaks up into short exact sequences, and that it is a sequence of modules for the graded ring $K_{*}(\mathbb{C} ; \mathbb{Z} / m)$. Since $K_{2 i}(E[s] ; \mathbb{Z} / m)=K_{2 i}(E ; \mathbb{Z} / m)$, this gives us a commutative diagram of graded modules with exact rows:

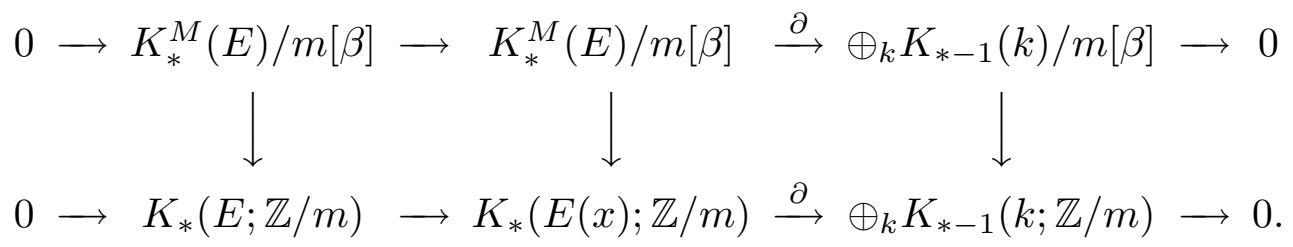

By assumption, the outside vertical maps are isomorphisms. The 5-lemma shows that the middle vertical is an isomorphism too.

Proof of theorem $A$. The ring map $K_{*}^{M}(F) \rightarrow K_{*}(F ; \mathbb{Z} / m)$ induces a ring map $\phi: K_{*}^{M}(F) / m[\beta] \rightarrow K_{*}(F ; \mathbb{Z} / m)$ sending the indeterminate $\beta$ to the Bott element in $K_{2}(F ; \mathbb{Z} / m)$. By theorem 3.1, the composition $\rho \phi$ is the natural injection

$$
K_{*}^{M}(F) / m[\beta] \subset K_{*}^{M}(F) / m\left[\beta, \beta^{-1}\right] \cong K_{*}^{e t}(F ; \mathbb{Z} / m) .
$$

Thus $\phi$ is an injection. We need to show that $\phi$ is onto. For this we use induction on $d=$ tr. deg. $(F)$. By proposition 2.4, there is a natural decomposition of $K_{n}(F ; \mathbb{Z} / m)$ as the direct sum of subgroups $\Phi_{n p}(F)$, each naturally isomorphic to $K_{p}^{M}(F) / m$, where $p \leq n$ and $p \equiv n(\bmod 2)$.

For $p<d$, the group $K_{p}^{M}(F)$ is generated by terms $\left\{x_{1}, \ldots, x_{p}\right\}$ where $p<d$. Since tr. deg. $\mathbb{C} \mathbb{C}\left(x_{1}, \ldots, x_{p}\right) \leq p, K_{p}^{M}(F)$ is generated by the images of the groups $K_{p}^{M}(E)$ as $E$ ranges over all subfields $E$ of $F$ having transcendence degree $\leq p$ over $\mathbb{C}$. Hence the corresponding subgroup $\Phi_{n p}(F)$ of $K_{n}(F ; \mathbb{Z} / m)$ is generated by the images of the subgroups $\Phi_{n p}(E)$. For each such $E$, our inductive hypothesis yields an isomorphism fitting into a commutative diagram of ring homomorphisms:

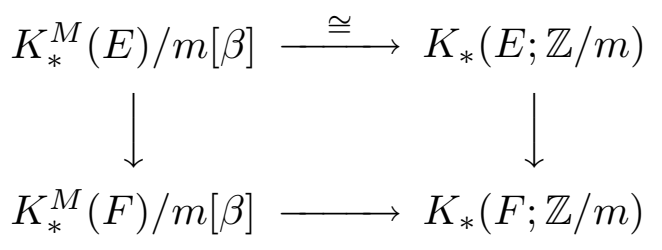


Hence the image of the bottom map contains every summand $\Phi_{n p}(F)$ for $p<d$.

It remains to consider the summands $\Phi_{n d}(F)$ of $K_{n}(F ; \mathbb{Z} / m)$; these are isomorphic to $H_{\text {et }}^{d}(F ; \mathbb{Z} / m) \cong K_{d}^{M}(F) / m$. This summand is generated by terms $a$ corresponding to $\left\{x_{1}, \ldots, x_{d}\right\}$, and such a term comes from an element $a_{0}$ of the summand $\Phi_{n d}\left(F_{0}\right)$ of $K_{n}\left(F_{0} ; \mathbb{Z} / m\right)$, where $F_{0}=\mathbb{C}\left(x_{1}, \ldots, x_{d}\right)$. Since $\Phi_{n d}\left(F_{0}\right)=0$ when tr. deg. $\left(F_{0}\right)<d$, we may assume that $F_{0}$ is a purely transcendental subfield of $F$. Write $F_{0}=E\left(x_{1}\right)$, where $E=\mathbb{C}\left(x_{2}, \ldots, x_{p}\right)$. By the inductive hypothesis, $K_{*}^{M}(k) / m[\beta] \cong K_{*}(k ; \mathbb{Z} / m)$ for all finite extensions $k$ of $E$. By lemma 3.3 the same is true for $F_{0}$. Hence $a_{0} \in \Phi_{n d}\left(F_{0}\right)$ comes from an element of $K_{*}^{M}\left(F_{0}\right)[\beta]$, whose image in $K_{*}^{M}(F)[\beta]$ maps to $a$. Hence the map is onto, as desired. 


\section{$\S 4$. The Quillen-Lichtenbaum Conjecture}

If $K U^{*}(X)$ denotes the topological $K$-theory of complex vector bundles on the underlying space $X(\mathbb{C})$, and $K U^{*}(X ; \mathbb{Z} / m)$ is the corresponding theory with coefficients $\mathbb{Z} / m$, then we know by $[\mathrm{Fr} 1,1.6]$ that $K_{n}^{e t}(X ; \mathbb{Z} / m) \cong K U^{-n}(X ; \mathbb{Z} / m)$.

Theorem 4.1. (Quillen-Lichtenbaum Conjecture for varieties over $\mathbb{C}$ ) Let $X$ be a smooth variety over $\mathbb{C}$. Assume either that (i) $m=2^{\nu}$ or (ii) the norm residue conjecture holds for $m$. Then the canonical isomorphism

$$
\rho_{n}(X): K_{n}(X ; \mathbb{Z} / m) \rightarrow K_{n}^{e t}(X ; \mathbb{Z} / m) \cong K U^{-n}(X ; \mathbb{Z} / m)
$$

is an isomorphism for all $n \geq \operatorname{dim}(X)-1$, and an injection for $n=\operatorname{dim}(X)-2$.

Suslin announced this result for curves and surfaces in [SuM, 4.7]; see [PW, 2.2]. Suslin's proof goes through in our setting, as it only depends upon our theorem A.

Proof. (Suslin) Using the $K$-theory localization sequence and induction on $d=$ $\operatorname{dim}(X)$, theorem A implies that the "Bott" $\operatorname{map} K_{n}^{\prime}(X ; \mathbb{Z} / m) \rightarrow K_{n+2}^{\prime}(X ; \mathbb{Z} / m)$ (multiplication by the Bott element) is an injection for $n=d-2$ and an isomorphism for $n \geq d-1$, where $X$ is any irreducible scheme over $\mathbb{C}$. When $X$ is smooth, so that $K_{n}(X ; \mathbb{Z} / m) \cong K_{n}^{\prime}(X ; \mathbb{Z} / m)$, this Bott periodicity implies the result by Thomason's theorem [T, 4.1] [DFST].

Remark 4.1.1. Levine uses [FS] to state this result more generally for varieties over fields of finite étale cohomological dimension in [L, 13.3].

Let $K_{n}^{r e l}(X)$ denote the relative term in the natural sequence

$$
\rightarrow K_{n+1}(X) \stackrel{\rho}{\rightarrow} K U^{-n-1}(X) \rightarrow K_{n}^{r e l}(X) \rightarrow K_{n}(X) \stackrel{\rho}{\rightarrow} K U^{-n}(X) \rightarrow
$$

Corollary 4.3. For $n \geq \operatorname{dim}(X)-2$, the groups $K_{n}^{r e l}(X)$ are uniquely 2-divisible. If the norm residue conjecture holds, the groups $K_{n}^{\text {rel }}(X)$ are uniquely divisible.

If $n=\operatorname{dim}(X)-3, K_{n}^{r e l}(X)$ is 2-torsionfree, and $\ell$-torsionfree for every other prime $\ell$ for which the norm residue conjecture holds.

Proof. Write $K_{n, \ell}^{r e l}$ for $K_{n}^{r e l}(X ; \mathbb{Z} / \ell)$. The usual homological yoga yields a sequence with coefficients $\mathbb{Z} / \ell$ :

$$
K_{n+1}(X ; \mathbb{Z} / \ell) \stackrel{\rho}{\rightarrow} K U^{-n-1}(X ; \mathbb{Z} / \ell) \rightarrow K_{n, \ell}^{r e l} \rightarrow K_{n}(X ; \mathbb{Z} / \ell) \stackrel{\rho}{\rightarrow} K U^{-n}(X ; \mathbb{Z} / \ell)
$$

When $n \geq \operatorname{dim}(X)-2$, the group $K_{n, \ell}^{r e l}$ vanishes by 4.1 . It contains $K_{n}^{r e l}(X) / \ell$ as a subgroup, and the $\ell$-torsion in $K_{n-1}^{r e l}(X)$ as a quotient, whence the result.

Now $K U^{-n}(X)$ is a finitely generated group; if $B_{n}$ is its torsion subgroup and $r_{n}$ its rank, then $K U^{-n}(X) \cong B_{n} \oplus \mathbb{Z}^{r_{n}}$. 
Theorem 4.4. For $n \geq \operatorname{dim}(X)-2, K_{n}(X)$ fits into an extension

$$
0 \rightarrow K_{n}^{0}(X) \rightarrow K_{n}(X) \rightarrow Q_{n} \rightarrow 0
$$

where $Q_{n}$ is a finitely generated group of rank $\sigma \leq r_{n},\left(Q_{n}\right)_{2 \text {-tors }}=K U^{-n}(X)_{2 \text {-tors }}$ and $K_{n}^{0}(X)$ is 2-divisible with 2-corank $\tau \leq r_{n+1}$. Thus if we localize at the prime 2 the group $K_{n}^{0}(X)$ becomes divisible and there is a uniquely divisible group $V_{n}$ such that

$$
K_{n}(X)_{(2)} \cong\left(B_{n}\right)_{(2)} \oplus \mathbb{Z}_{(2)}^{\sigma} \oplus(\mathbb{Q} / \mathbb{Z})_{(2)}^{\tau} \oplus V_{n}
$$

If the norm residue conjecture holds, $K_{n}^{0}(X)$ is divisible with torsion subgroup $(\mathbb{Q} / \mathbb{Z})^{\tau}, \tau \leq r_{n+1}$, and $Q_{n}=B_{n} \oplus \mathbb{Z}^{\sigma}$ for some $\sigma \leq r_{n}$. Thus there is a uniquely divisible group $V_{n}$ such that

$$
K_{n}(X) \cong B_{n} \oplus \mathbb{Z}^{\sigma} \oplus(\mathbb{Q} / \mathbb{Z})^{\tau} \oplus V_{n}
$$

Proof. The first assertion is obvious from (4.2): $K_{n}^{0}(X)=\operatorname{ker}(\rho)$ and $Q_{n}=\operatorname{im}(\rho)$. The rest is obvious from 4.3 , since after localizing at 2 the group $K_{n}^{r e l}(X)$ becomes uniquely divisible $\left(\cong \mathbb{Q}^{\tau} \oplus V_{n}\right)$ and $K_{n-1}^{r e l}(X)$ becomes torsionfree.

Remark 4.4.1. We will see in theorem $\mathrm{C}$ that when $n>\operatorname{dim}(X)$ we have $\sigma=0$ and when $n \geq \operatorname{dim}(X)$ we have $\tau=r_{n+1}$. This can be improved somewhat when $X$ is projective. However in the example $X=\operatorname{Spec} \mathbb{C}\left[x, x^{-1}, y, y^{-1}\right]$ we have $\sigma=1$ for $n=\operatorname{dim}(X)$, and $1=\tau<r_{n+1}=2$ for $n=\operatorname{dim}(X)-1$; see [PW, 6.8.1].

Another interesting case of theorem 4.4 arises when $n=0$ and $X$ is an irreducible projective surface. Here $K_{0}(X) \cong \mathbb{Z} \oplus \operatorname{Pic}(X) \oplus C H^{2}(X)$, the Picard group $\operatorname{Pic}(X)$ is an extension of the finitely generated Néron-Severi group $N S(X)$ by the divisible $\operatorname{Pic}^{0}(X)$, and the Chow group $C H^{2}(X)$ is an extension of the degree part $\mathbb{Z}$ by the divisible group $A_{0}(X)$ of zero-cycles of degree zero. From this it is easy to see that in the decomposition of theorem 4.4 we have $Q_{0}=\mathbb{Z}^{2} \oplus N S(X)$, while $K_{0}^{0}(X)$ is the sum of the divisible groups $\operatorname{Pic}^{0}(X)$ and $A_{0}(X)$. 


\section{$\S 5$. Divisibility of $K_{*}(F)$}

We now turn to theorem B.

Theorem B (5.1). Let $F$ be a field of transcendence degree $d$ over $\mathbb{C}$. Then the groups $K_{n}(F)$ are 2-divisible for all $n>d$.

If the norm residue conjecture holds for $F$ then the groups $K_{n}(F)$ are divisible for all $n>d$.

Of course, this implies that the torsion subgroup of $K_{n}(F)$ is isomorphic to the divisible group $K_{n+1}(F, \mathbb{Q} / \mathbb{Z})$, which was described in 2.4 and 1.2 .

We will need the Deligne-Beilinson cohomology groups $H_{\mathcal{D}}^{*}$, and we refer the reader to $[\mathrm{EV}]$ for details of their construction. We shall also need the Chern class maps, which were constructed in [Bei] and [Gi1, Gi2].

$$
c_{i}: K_{n}(X) \rightarrow H_{\mathcal{D}}^{2 i-n}(X, \mathbb{Z}(i)), \quad i \geq 1
$$

Examples 5.2. If $a \in F^{\times}$, then $c_{n}(a)=0$ for $n \neq 1$, and $c_{1}(a)$ is the image of $a$ under the isomorphism $H_{\mathcal{D}}^{1}(F, \mathbb{Z}(1)) \cong F^{\times}$.

If $a_{1}, \ldots, a_{j}$ are units in $F$, and $x=\left\{a_{1}, \ldots, a_{j}\right\} \in K_{j}(F)$, write $(x)$ for the cup product $c_{1}\left(a_{1}\right) \cdots c_{1}\left(a_{j}\right)$ in $H_{\mathcal{D}}^{j}(F, \mathbb{Z}(j))$. Then the product formula yields $c_{j}(x)=(-1)^{j-1}(j-1) !(x)$, and $c_{n}(x)=0$ for $n \neq j$.

Similarly, the choice of an $m$ th root of unity $\zeta \in \mathbb{C}^{\times}$determines a unique Bott element $\beta \in K_{2}(\mathbb{C} ; \mathbb{Z} / m)$ whose Bockstein is $\zeta$, and elements $\zeta(i) \in H_{\mathcal{D}}^{1}(\mathbb{C}, \mathbb{Z}(i)) \cong$ $\mathbb{C}^{\times}(i), i \geq 1$. Let $\gamma_{i} \in{ }_{m} K_{2 i-1}(\mathbb{C})$ denote the Bockstein of $\beta^{i} \in K_{2 i}(\mathbb{C}, \mathbb{Z} / m)$. It was proven in $[\mathrm{PW}, 4.4]$ that $c_{i}\left(\gamma_{i}\right)$ is $(-1)^{i-1}(i-1) ! \zeta(i)$, and the proof there shows that $c_{n}\left(\gamma_{i}\right)=0$ for $n \neq i$.

Lemma 5.3. Let $F=\mathbb{C}(X)$ be the field of rational functions of a smooth variety $X$ over $\mathbb{C}$. Assume that either ( $i) \ell=2$ or (ii) the norm residue conjecture holds for $\ell$. Then for all $m=\ell^{\nu}$ :

(1) ${ }_{m} H_{\mathcal{D}}^{j}(X, \mathbb{Z}(i)) \cong{ }_{m} H_{\text {an }}^{j-1}\left(X, \mathbb{C}^{\times}\right)$for all $i \geq j$;

(2) ${ }_{m} H_{\mathcal{D}}^{j}(F, \mathbb{Z}(i)) \cong{ }_{m} H_{\text {an }}^{j-1}\left(F, \mathbb{C}^{\times}\right) \cong H_{\text {et }}^{j-1}(F, \mathbb{Z} / m)$ for all $i \geq j$;

Proof. (Cf. [PW, 4.1]) Suppose $i \geq j$, so that $F^{i} H_{\text {an }}^{j-1}(X, \mathbb{C})=0$. There is an exact sequence (see [EV, 2.10.c]):

$$
0 \rightarrow H_{\mathrm{an}}^{j-1}\left(X, \mathbb{C}^{\times}(i)\right) \rightarrow H_{\mathcal{D}}^{j}(X, \mathbb{Z}(i)) \rightarrow F^{i} H_{\mathrm{an}}^{j}(X, \mathbb{C})
$$

Since $F^{i} H^{j}(X, \mathbb{C})$ is torsionfree, this yields part (1). Part (2) follows from 1.4(1) and (1) by taking the limit over all open $U$ in $X$.

Remark 5.3.1. Let $a_{1}, \ldots, a_{j}$ be units in $F$, and $x=\left\{a_{1}, \ldots, a_{j}\right\} \in K_{j}(F)$. For $i \geq 1$ and $q=i+j$, the isomorphism $K_{j}^{M}(F) / m \cong H_{\mathrm{et}}^{j}(F, \mathbb{Z} / m(j)) \cong$ $H_{\text {et }}^{j}(F, \mathbb{Z} / m(q))$ sends $x$ to $\zeta^{\otimes i} \otimes\left(a_{1}\right) \cup \cdots \cup\left(a_{j}\right)$; the isomorphism $H_{\text {et }}^{j}(F, \mathbb{Z} / m(q)) \cong$ ${ }_{m} H_{\mathcal{D}}^{j+1}(F, \mathbb{Z}(q))$ in $5.3(2)$ sends this element to $\zeta(i) \cup(x)$. 
Now recall from theorem A that every element of $K_{n}(F, \mathbb{Z} / m)$ is a sum of terms $\beta^{i} x_{j}$, where $x_{j} \in K_{j}^{M}(F) / m$ and $2 i+j=n$. The Bockstein $\partial$ sends $\beta^{i} x_{j}$ to the $K$-theory product $\left\{\gamma_{i}, x_{j}\right\}$ in $K_{n-1}(F)$, where $\gamma_{i}=\partial\left(\beta^{i}\right) \in K_{2 i-1}(\mathbb{C})$. Thus every element in the image of $K_{n}(F, \mathbb{Z} / m) \rightarrow K_{n-1}(F)$ may be written as a sum $x=\sum_{2 i+j=n}\left\{\gamma_{i}, x_{j}\right\}$.

Lemma 5.4. For each element $x=\sum_{2 i+j=n}\left\{\gamma_{i}, x_{j}\right\}$ in ${ }_{m} K_{n-1}(F)$ and each $q<n$, the Chern class

$$
{ }_{m} K_{n-1}(F){\stackrel{c_{q}}{\longrightarrow}}_{m} H_{\mathcal{D}}^{2 q+1-n}(F, \mathbb{Z}(q)) \cong H_{\mathrm{et}}^{2 q-n}(F, \mathbb{Z} / m) \cong K_{2 q-n}^{M}(F) / m
$$

sends $x$ to $(-1)^{q-1}(q-1) ! x_{2 q-n}$.

Proof. The hypothesis $q<n$ implies that $q \geq 2 q+1-n$. The displayed isomorphism between ${ }_{m} H_{\mathcal{D}}^{2 q+1-n}(F, \mathbb{Z}(q))$ and $H_{\text {et }}^{2 q-n}(F, \mathbb{Z} / m)$ is now given by lemma 5.3 .

The product formula for $c_{q}$ ([Gi2, p. 229][Sch, p. 28]) and example 5.2 shows that $c_{q}\left(\left\{\gamma_{i}, x_{j}\right\}\right)=0$ for $q \neq i+j$, and that if $q=i+j$ then in $H_{\mathcal{D}}^{2 q+1-n}(F, \mathbb{Z}(q))$ :

$$
c_{q}\left(\left\{\gamma_{i}, x_{j}\right\}\right)=\frac{-(q-1) !}{(i-1) !(j-1) !} c_{i}\left(\gamma_{i}\right) \cup c_{j}\left(x_{j}\right)=(-1)^{q-1}(q-1) ! \zeta(i) \cup\left(x_{j}\right)
$$

Since $q$ determines $i=n-q$ and $j=2 q-n$, there is only one such term in $c_{q}(x)$. The result now follows from remark 5.3.1, which states that the isomorphism $K_{j}(F) / m \cong{ }_{m} H_{\mathcal{D}}^{j+1}(F, \mathbb{Z}(q))$ sends $x_{j}$ to $\zeta(i) \cup\left(x_{j}\right)$.

Proof of theorem B. Suppose that $K_{n}(F)$ is not $\ell$-divisible for some $n$, and choose $x \in K_{n}(F)-\ell K_{n}(F)$. For each $m=\ell^{\nu}$, the image $\bar{x}$ of $x$ in $K_{n}(F) / m \subseteq$ $K_{n}(F, \mathbb{Z} / m)$ is not in $\ell \cdot K_{n}(F, \mathbb{Z} / m)$, so it is an element of exponent exactly $m$. By theorem A we can write $\bar{x}=\sum \beta^{i} x_{j}$ where $x_{j} \in K_{j}^{M}(F) / m$ and $2 i+j=$ $n$. Pick $j$ so that $x_{j} \neq \ell \cdot K_{j}^{M}(F)$ and set $q=i+j$. By construction of $\bar{x}$, $\sum_{2 i+j=n}\left\{\gamma_{i}, x_{j}\right\}=\partial(\bar{x})=0$ in ${ }_{m} K_{n-1}(F)$. But $c_{q}(\partial \bar{x})= \pm(q-1) ! x_{j}$ is nonzero for $\nu \gg 0$. This contradiction proves that $K_{n}(F)$ is $\ell$-divisible. 


\section{$\S 6$. THEOREM C}

In this section we shall prove theorem $\mathrm{C}$, that $K_{n}(X)$ is divisible-by-finite when $n>\operatorname{dim}(X)$, provided that we localize at a prime $\ell$ for which the norm residue conjecture holds (such as $\ell=2$ ). Since our technique is to compare with the finitely generated groups $H_{\text {an }}^{j}(X, \mathbb{Z})$ and $K U^{-n}(X)$, it is convenient to write $A_{j}=$ $A_{j}(X)$ and $B_{n}=B_{n}(X)$ for the torsion subgroups of $H_{\mathrm{an}}^{j}(X, \mathbb{Z})$ and $K U^{-n}(X)$, respectively. Thus

$$
H_{\mathrm{an}}^{j}(X, \mathbb{Z}) \cong A_{j} \oplus \mathbb{Z}^{b_{j}} \text { and } K U^{-n}(X) \cong B_{n} \oplus \mathbb{Z}^{r_{n}},
$$

where $b_{j}$ is the $j$ th Betti number of $X$, and $r_{n}=\sum_{i \geq n / 2}^{\infty} b_{2 i-n}$. We shall also write $V_{n}$ for the uniquely divisible group $V_{n}=K_{n}^{0}(X) \otimes \mathbb{Q}$, and $\mathbb{Q}_{\ell} / \mathbb{Z}_{\ell}$ for the $\ell$-primary subgroup of $\mathbb{Q} / \mathbb{Z}$. The localization of an abelian group $K$ at the prime $\ell$ will be written as $K_{(\ell)}$.

Theorem C (6.1). Let $X$ be a smooth variety over $\mathbb{C}$. Suppose that $n$ satisfies either (a) $n>\operatorname{dim}(X)$ or (b) $X$ is projective and $n \geq \max \{1, \operatorname{dim}(X)-2\}$. If the norm residue conjecture holds then $K_{n}(X)$ is a divisible-by-finite group, with

$$
K_{n}(X) \cong B_{n} \oplus(\mathbb{Q} / \mathbb{Z})^{r_{n+1}} \oplus V_{n}
$$

In general, this isomorphism exists after localizing at any prime $\ell$ (such as $\ell=2$ ) for which the norm residue conjecture holds. That is, for such an $\ell$ and for all $n$ satisfying (a) or (b) above the localization $K_{n}(X)_{(\ell)}$ is divisible-by-finite, and there is a noncanonical decomposition

$$
K_{n}(X)_{(\ell)} \cong B_{n(\ell)} \oplus\left(\mathbb{Q}_{\ell} / \mathbb{Z}_{\ell}\right)^{r_{n+1}} \oplus V_{n}
$$

Here $B_{n(\ell)}$ is the $\ell$-torsion subgroup of $K U^{-n}(X), r_{n+1}$ is the rank of $K U^{-n-1}(X)$, and $V_{n}$ is uniquely divisible.

If either $\left(a^{\prime}\right) n=\operatorname{dim}(X)$ or $\left(b^{\prime}\right) X$ is projective and $n=\max \{0, \operatorname{dim}(X)-2\}$, we still have $K_{n}(X)_{\ell \text {-tors }} \cong B_{n(\ell)} \oplus\left(\mathbb{Q}_{\ell} / \mathbb{Z}_{\ell}\right)^{r_{n+1}}$.

Example 6.1.1. If $X$ is a smooth projective surface over $\mathbb{C}$, this follows for all $n>0$ from $[\mathrm{PW}, 6.7]: K_{n}(X) \cong B_{n} \oplus(\mathbb{Q} / \mathbb{Z})^{r_{n+1}} \oplus V_{n}$. In this case, $B_{n}$ is either $A_{2}$ or $A_{3}$, depending on the parity of $n$. For $n=0$, we saw in 4.4.1 that the image of $K_{0}(X) \rightarrow K U^{0}(X)$ is $\mathbb{Z}^{2} \oplus N S(X)$, where the Néron-Severi group is $N S(X)=A_{2} \oplus \mathbb{Z}^{\rho}$. In this case $K_{0}(X) \cong \mathbb{Z}^{2+\rho} \oplus A_{2} \oplus(\mathbb{Q} / \mathbb{Z})^{r_{1}} \oplus V_{0}$, where $r_{1}=b_{1}+b_{3}=4 q$, where $q=\operatorname{dim} H^{1}\left(X, \mathcal{O}_{X}\right)$; see [PW, 6.3].

Example 6.1.2. If $X$ is a smooth projective 3-fold over $\mathbb{C}$, then theorem $\mathrm{C}$ holds for all $n \geq 1$. The lack of divisibility information about $K_{0}^{\text {rel }}(X)$ (see 4.3) prevents us from making any blanket assertion about $K_{0}(X)_{\text {tors }}$. Nevertheless, we can say something here in terms of the Chow groups $C H^{i}(X)$ of codimension $i$ cycles modulo rational equivalence.

Now $C H^{3}(X)$ is the sum of a divisible group and $\mathbb{Z}$, and Roitman's theorem states that $C H^{3}(X)_{\text {tors }} \cong \operatorname{Alb}(X)_{\text {tors }} \cong(\mathbb{Q} / \mathbb{Z})^{2 q}$. The composition of the cycle 
map $C H^{3}(X) \rightarrow K_{0}(X)$ with the Chern class $c_{3}: K_{0}(X) \rightarrow C H^{3}(X)$ is multiplication by 2 , so the image $F^{3} K_{0}(X)$ of $C H^{3}(X)$ in $K_{0}(X)$ is isogenous to $C H^{3}(X)$. The composition of the cycle map with the direct image $K_{0}(X) \rightarrow K_{0}(\mathbb{C})=\mathbb{Z}$ coincides with the projection $C H^{3}(X) \rightarrow \mathbb{Z}$, so $F^{3} K_{0}(X)$ is a direct summand of $K_{0}(X)$. Since $c_{1}$ and $c_{2}$ vanish on $F^{3} K_{0}(X)$, we have a decomposition

$$
K_{0}(X) \cong \mathbb{Z} \oplus \operatorname{Pic}(X) \oplus C H^{2}(X) \oplus F^{3} K_{0}(X) .
$$

As with surfaces, the torsion in $N S(X)$ is $A_{2}$, so $\operatorname{Pic}(X)_{\text {tors }}$ is $A_{2} \oplus(\mathbb{Q} / \mathbb{Z})^{2 q}$. By $[\mathrm{Su} 2], C H^{2}(X)_{\text {tors }}$ is $N H_{\mathrm{et}}^{3}(X, \mathbb{Q} / \mathbb{Z})$, the kernel of $H_{\mathrm{et}}^{3}(X, \mathbb{Q} / \mathbb{Z}) \rightarrow H_{\mathrm{et}}^{3}(F, \mathbb{Q} / \mathbb{Z})$, where $F=\mathbb{C}(X)$. Assembling this information, we obtain:

$$
K_{0}(X)_{\text {tors }} \cong A_{2} \oplus(\mathbb{Q} / \mathbb{Z})^{4 q} \oplus N H_{\mathrm{et}}^{3}(X, \mathbb{Q} / \mathbb{Z})
$$

Note that $A_{4}$ is a summand of $N H_{\text {et }}^{3}(X, \mathbb{Q} / \mathbb{Z})$; this follows from 1.1 and the calculation that $H_{\text {et }}^{3}(X, \mathbb{Q} / \mathbb{Z}) \cong A_{4} \oplus(\mathbb{Q} / \mathbb{Z})^{b_{3}}$.

The proof of theorem $\mathrm{C}$ will use Beilinson's observation [Bei, 1.7.3] that the Deligne Chern classes $c_{i}$ are compatible with the analytic Chern classes. That is, if we set $n+j=2 i$ then the following diagram commutes, with the bottom row exact:

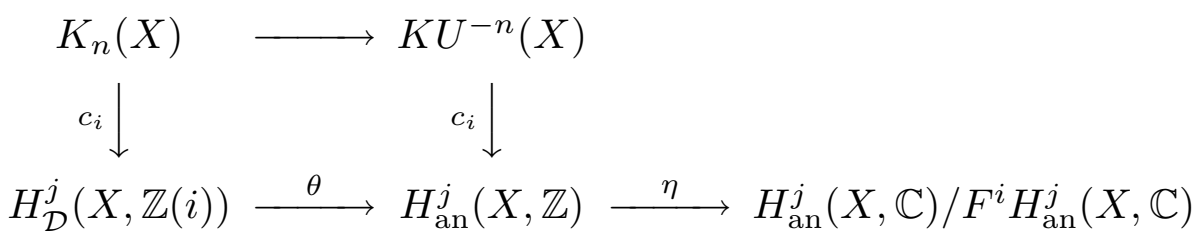

Lemma 6.2. Let $X$ be a smooth complex variety of dimension $d$, and set $n=$ $2 i-j$. Then the image of $\theta$ is the torsion subgroup $A_{j}$ of $H_{\mathrm{an}}^{j}(X, \mathbb{Z})$, provided that either (a) $n>\operatorname{dim}(X)$, or (b) $X$ is projective and $n>0$.

Proof. (a) By [D, 8.2.4], $F^{i} H_{\mathrm{an}}^{j}(X, \mathbb{C})=0$ if $i>\operatorname{dim}(X)$ or if $i>j$. If $i \leq j$ and $i \leq d$ then $n=2 i-j \leq i \leq d$. Thus if $n>\operatorname{dim}(X)$ then $\eta$ is the complexification $\operatorname{map} H_{\mathrm{an}}^{j}(X, \mathbb{Z}) \rightarrow H_{\mathrm{an}}^{j}(X, \mathbb{C})$. Hence $\operatorname{im}(\theta)=$ ker $\eta$ is the torsion subgroup $A_{j}$.

Part (b) is due to Gillet $[\mathrm{Gi} 2,5.5]$. When $X$ is a smooth projective complex variety, the Hodge structure on $H_{\mathrm{an}}^{j}(X, \mathbb{Z})$ is pure of weight $j$. If $n>0$ then $j<2 i$ and we have $H_{\text {an }}^{j}(X, \mathbb{R}) \cap F^{i} H_{\text {an }}^{j}(X, \mathbb{C})=0$. Therefore the kernel of $\eta$ is the kernel $A_{j}$ of $H_{\text {an }}^{j}(X, \mathbb{Z}) \rightarrow H_{\text {an }}^{j}(X, \mathbb{R})$. But $A_{j}$ is the image of $\theta: H_{\mathcal{D}}^{j}(X, \mathbb{Z}(i)) \rightarrow$ $H_{\mathrm{an}}^{j}(X, \mathbb{Z})$.

Remark 6.2.1. This fails for $n=d$, for example if $X=\operatorname{Spec} \mathbb{C}\left[t_{1}, t_{1}^{-1}, \ldots t_{d}, t_{d}^{-1}\right]$.

The next lemma is well known (cf. [Fr2, 3.4][Bei, 2.3]), but we were unable to find a good reference for it. Note that as stated it fails for $n=0$, but it would hold for $n=0$ if we replaced the non-existent $c_{0}$ with the rank function.

Lemma 6.3. For every $n>0$ :

a) $K U^{-n}(L) \otimes \mathbb{Q} \stackrel{\oplus c_{i}}{\longrightarrow} \oplus_{i} H_{\mathrm{an}}^{2 i-n}(L, \mathbb{Q})$ is an isomorphism for any compact space $L$; b) $K U^{-n}(X) \otimes \mathbb{Q} \stackrel{\oplus c_{i}}{\longrightarrow} \oplus_{i} H_{\mathrm{an}}^{2 i-n}(X, \mathbb{Q})$ is an isomorphism for any complex variety $X$. 
Proof. Write $X=\bar{X}-Z$ for compact $\bar{X}$ and $Z$. If $D$ is a small tubular neighborhood of $Z_{\text {an }}$ then $L=\bar{X}_{\text {an }}-D$ is compact and $K U^{*}(X) \cong K U^{*}(L)$. So it suffices to prove that $K U^{-n}(L) \otimes \mathbb{Q} \cong \oplus_{i} H_{\text {an }}^{2 i-n}(L, \mathbb{Q})$ for any compact space $L$.

If $n=0,-1$ this is classical, except that the rank is used in place of the nonexistent $c_{0}$; see $[\mathrm{K}, \mathrm{V} .3 .26]$. For $n \leq-2$, the Bott periodicity element $\beta \in$ $K U^{-2}$ (point) yields the formula $c_{i+1}(x \cdot \beta)=-i \cdot c_{i}(x)$ for all $x \in K U^{-n+2}(L)$ when $i>0$, and $c_{1}(x \cdot \beta)=\operatorname{rank}(x)$ for $x \in K U^{0}(L)$. The result now follows from induction on $n$.

Corollary 6.4. Assume that either (a) $n>\operatorname{dim}(X)$ or (b) $X$ is projective, and $n \geq \max \{1, \operatorname{dim}(X)-2\}$. Then the image of $\rho_{n}: K_{n}(X) \rightarrow K U^{-n}(X)$ is finite, and $B_{n} /$ im $\left(\rho_{n}\right)$ is a finite group of odd order, whose order is prime to every prime $\ell$ for which the norm residue conjecture holds.

Proof. Form the commutative diagram

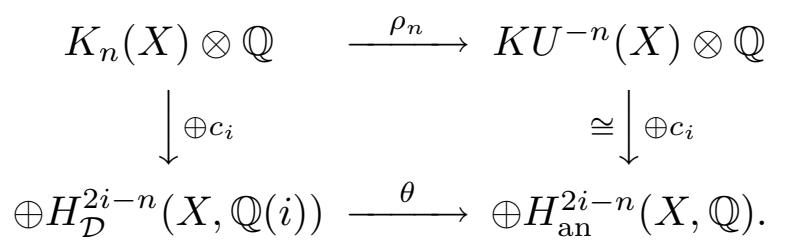

By lemma 6.3 the right vertical map is an isomorphism. By lemma 6.2, the bottom map is zero when either (a) or (b) holds. Hence the top map is zero, i.e., the image of $\rho_{n}$ is contained in $B_{n}$. Since the cokernel lies in $K_{n-1}^{r e l}(X)$, which has no $\ell$-torsion when $n \geq \operatorname{dim}(X)-2$ by 4.3 , the rest follows.

Proof of theorem $C$. Set $d=\operatorname{dim}(X)$. By theorem 4.4, we have a decomposition for all $n \geq d-2$ :

$$
K_{n}(X)_{(\ell)} \cong B_{n(\ell)} \oplus \mathbb{Z}_{(\ell)}^{\sigma} \oplus\left(\mathbb{Q}_{\ell} / \mathbb{Z}_{\ell}\right)^{\tau} \oplus V_{n}
$$

Here $\sigma$ is the rank of $\rho_{n}$ and $\tau$ is $r_{n+1}-\operatorname{rank}\left(\rho_{n+1}\right)$. When (a) or (b) holds, the images of $\rho_{n}$ are finite by 6.4 , so their ranks $\sigma$ and $r_{n+1}-\tau$ must be zero.

When $n=d$, or $X$ is projective and $n=\max \{0, d-2\}$, the image of $\rho_{n+1}$ is still finite by 6.4 , so $r_{n+1}-\tau$ must still be zero, i.e., $\tau$ must equal $r_{n+1}$.

Finally, if the norm residue conjecture holds we may replace (6.5) by the decomposition $K_{n}(X) \cong B_{n} \oplus \mathbb{Z}^{\sigma} \oplus(\mathbb{Q} / \mathbb{Z})^{\tau} \oplus V_{n}$ of theorem 4.4. 


\section{REFERENCES}

[AM] M. Atiyah and I. Macdonald, Introduction to commutative algebra, Addison-Wesley, Reading, Mass., 1969.

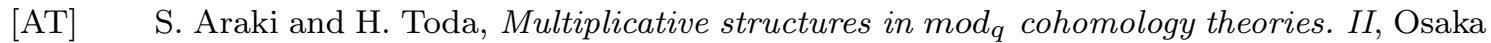
J. Math. 3 (1966), 81-120.

[Bei] A. Beilinson, Higher regulators and values of L-functions, Itogi Nauki i Tekhniki 24 (1984), 181-238. (Russian)

[Bl] S. Bloch, Algebraic cycles and higher K-theory, Adv. Math. 61 (1986), 267-304.

[BK] S. Bloch and K. Kato, p-adic étale cohomology, Publ. Math. IHES 63 (1986), 107-152.

[BL] S. Bloch and S. Lichtenbaum, A spectral sequence for motivic cohomology, preprint (1994), to appear in Invent. Math..

[BV1] L. Barbieri-Viale, Cicli di codimensione 2 su varietà unirationali complesse, Astérisque, vol. 226, 1994, pp. 13-41.

[BV2] - On the Deligne-Beilinson cohomology sheaves, Preprint (1994) Alg. Geom. /9412006.

[CT-R] J.-L. Colliot-Thélène and W. Raskind, $\mathcal{K}_{2}$-Cohomology and the second Chow group, Math. Ann. 270 (1985), 165-199.

[D] P. Deligne, Théorie de Hodge III, Publ. Math. IHES (Paris) 44 (1974), 5-77.

[DF] W. Dwyer and E. Friedlander, Algebraic and étale K-theory, Trans. AMS 292 (1985), 247-280.

[DFST] W. Dwyer, E. Friedlander, V. Snaith and R. Thomason, Algebraic K-theory eventually surjects onto topological K-theory, Invent. Math. 66 (1982), 481-491.

[EV] H. Esnault and E. Viehweg, Deligne-Beilinson cohomology, Beilinson's conjectures on special values of $L$-functions, Perspect. Math. vol. 4, Academic Press, 1988, pp. 43-91.

[Fr1] E. Friedlander, Etale K-theory I: Connections with Etale Cohomology and Algebraic Vector Bundles, Invent. Math. 60 (1980), 105-134.

[Fr2] Etale K-theory II: connections with algebraic K-theory, Ann. Sc. École Norm. 15 (1982), 231-256.

[FS] E. Friedlander and A. Suslin, The spectral sequence relating algebraic K-theory to motivic cohomology, preliminary draft (1999) http://www.math.uiuc.edu/K-theory/0360.

[FV] E. Friedlander and V. Voevodsky, Bivariant cycle cohomology, Preprint (1995), Cycles, transfers and motivic homology theories, Princeton University Press, 2000, pp. 138-187.

[Gi1] H. Gillet, Deligne homology and Abel-Jacobi maps, Bull. AMS 10 (1984), 285-288.

[Gi2] - On the K-theory of surfaces with multiple curves and a conjecture of Bloch, Duke Math. J. 51 (1984), 195-233.

[GS] H. Gillet and C. Soulé, Filtrations on Higher K-theory, Proc. Symp. Pure Math., Proc. 1997 Seattle $K$-theory Conference, vol. 67, AMS, 1999, pp. 89-148.

[K] M. Karoubi, K-theory. An introduction, Springer Verlag, 1978.

[L] M. Levine, K-theory and motivic cohomology of schemes, preprint (1999), posted at http://www. math.uiuc.edu/K-theory/0336.

[MS] A. Merkurjev and A. Suslin, K-Cohomology of Severi-Brauer varieties and the norm residue homomorphism, Izv. Akad. Nauk. USSR 46 (1982), 1011-1046 (Russian); transl., Math. USSR Izvestiya 21 (1983), 307-340.

[PW] C. Pedrini and C. Weibel, The higher K-theory of a complex surface, Preprint (1998).

[RW] J. Rognes and C. Weibel, Two-primary algebraic K-theory of rings of integers in number fields, Jour. AMS 13 (2000), 1-54.

[Sch] P. Schneider, Introduction to the Beilinson conjectures, Beilinson's conjectures on special values of $L$-functions, Perspect. Math. vol. 4, Academic Press, 1988, pp. 1-35.

[Sou1] C. Soulé, K-théorie des anneaux d'entiers de corps de nombres et cohomologie étale, Invent. Math. 55 (1979), 251-295.

[Sou2] - Opérations en K-théorie, Canad. J. Math. 27 (1985), 488-550.

[Sou3] _ Letter to Bloch and Lichtenbaum, Feb. 8, 1995. 
[Su1] A. Suslin, On the K-theory of algebraically closed fields, Invent. Math. 73 (1983), $241-245$.

[Su2] —, Torsion in $K_{2}$ of fields, K-theory 1 (1987), 5-29.

[Su3] - Higher Chow groups and étale cohomology, Preprint (1994), Cycles, transfers and motivic homology theories, Princeton University Press, 2000, pp. 239-254.

[SuM] A. Suslin, Algebraic K-theory and Motivic Cohomology, Proc. 1994 I.C.M. Zürich, Birkhäuser, 1995, pp. 342-351.

[SV] A. Suslin and V. Voevodsky, Bloch-Kato conjecture and motivic cohomology with finite coefficients, Preliminary version 1995, The Arithmetic and Geometry of Algebraic Cycles, Kluwer, 2000, pp. 117-189.

[T] R. Thomason, Algebraic K-theory and étale cohomology, Ann. Sci. Ec. Norm. Sup. (Paris) 18 (1985), 437-552.

[V1] V. Voevodsky, Triangulated categories of motives over a field, Preprint (1995), Cycles, transfers and motivic homology theories, Princeton University Press, 2000, pp. 188-238.

[V2] V. Voevodsky, The Milnor Conjecture, Preprint (1997).

[W] C. Weibel, Products in higher Chow groups and motivic cohomology, Proc. Symp. Pure Math., Proc. 1997 Seattle K-theory Conference, vol. 67, AMS, 1999, pp. 305-315.

[W2] Étale Chern classes at the prime 2, Algebraic $K$-theory and Algebraic Topology, NATO ASI Series C, vol. 407, Kluwer Press, 1993.

Dima - Università di Genova, Genova, Italy

E-mail address: pedrini@dima.unige.it

Department of Mathematics, Rutgers University, New Brunswick, NJ U.S.A.

E-mail address: weibel@math.rutgers.edu 